\title{
Detection of Minimum Geometrical Variation by Free Space Based Chipless Approach and its Application to Authentication
}

\author{
Z. Ali, N. Barbot, R. Siragusa, E. Perret, Senior Member IEEE, D. Hely, M. Bernier, and F. Garet
}

\begin{abstract}
A free space based chipless approach using a second order bandpass filter model is presented in this article to detect minimum geometrical variations along the dimensions of a $\mathrm{C}$ folded scatterer. With the help of this model, further randomness inherent in fabrication process can also be analyzed and forecasted. Quad C-folded scatterers are used to realize chipless tags and three groups of tags exhibiting distinct arms length are fabricated. A cosine similarity function is then used to evaluate the similarity rate of electromagnetic signatures reflected by tags coming from different groups. Experimental results performed in a realistic outdoor environment, show that it is possible to discriminate two tags which differ from each other by a mean variation of around $25 \mu \mathrm{m}$ along the arms length. Finally, the proposed analytical model is capable to dissociate the tags by using merely two extracted independent quantities: the frequency of resonance and the quality factor.
\end{abstract}

Index Terms - anti-counterfeiting, authentication technology, chipless RFID, fabrication tolerance, microwave, randomness.

\section{INTRODUCTION}

$\mathrm{R}$ ELIABLE authentication methods have become a global demand to limit counterfeiting, which is widely spread in many consumer and nonconsumer industries. Although, conventional optical barcodes are prevalent in item level tagging, it is impossible to conduct authentication merely by them. At present, microchips along with magnetic tape are being utilized for unitary authentication in various applications such as bank cards, access control badges, and so on

Recently, radio frequency (RF) authentication based on randomness inherent in fabrication process, also appears to be an emerging technology for secure unitary authentication applications. For example, a chipless RF Identification (RFID) system for secure near field data transfer, especially for identification of bank cards, is presented in [1]. In this identification system, cavity measurements are used to discriminate the chipless tags by noting the frequency shifts of the resonant modes of the cavity. In [2], a sensitivity model

This work was supported in part by the University of Grenoble Alpes via the AGIR program and the Région Auvergne-Rhône-Alpes via the ARC6 program and in part by the Institut Universitaire de France.

Z. Ali, N. Barbot, R. Siragusa, E. Perret, and D. Hely are with the Univ. Grenoble Alpes, Grenoble INP, LCIS, 26000 Valence, France (e-mail: zeshan.ali@lcis.grenoble-inp.fr). E. Perret is also with the Institut Universitaire de France, Paris, France. M. Bernier and F. Garet are with the IMEP-LAHC - University of Savoie Mont Blanc, Chambery, France. using slot resonators is proposed, where variations along numerous slot parameters (air gap, trace width, substrate thickness, and dielectric constant of substrate) are considered.

In previous recent work [3], the discrimination of chipless tags signature based on geometrical variations of less than $10 \mu \mathrm{m}$ has been introduced. A proper analytical treatment has been presented to quantify very slight variations of geometrical dimensions of a $\mathrm{C}$-folded scatterer, where the varied geometrical dimensions are not directly linked to the frequency of resonance of the tag.

In this study, variations of the geometrical dimensions of Cfolded resonators are modeled and detected by using a second order bandpass filter model. The paper is organized as follows: Section II introduces the chipless tags and a mathematical model. Section III describes a 2D map of similarity produced by the model, where the theoretical similarities are related to the measurements. Subsequently, authentication results are presented and a comparison between the model and measurements is analyzed in section IV to validate the proposal, before concluding in section $\mathrm{V}$.

\section{Design OF THE ChIPLeSS TAGS AND AN ALTERNATIVE MODEL FOR C-FOLDED SCATTERER}

The C-folded dipole design and behavior have been discussed in [4], [5]. The frequency of resonance of such scatterer can be calculated using the expression found in [4]:

$$
f_{r}=\frac{c}{4 L^{\prime} \sqrt{\varepsilon_{e f f}}}
$$

where, $L^{\prime}$ is the total length of the arms (i.e. summation of the effective length $L$ and supplementary length $\Delta L$ added to compensate the fringing field effects at both the open- and short-circuited ends of the scatterer), and $\varepsilon_{e f f}$ is the effective relative permittivity of the coplanar stripline, which depends on the gap value $g$, the width $w$, the thickness $t$ of the strips, the substrate thickness $h$, and its permittivity $\varepsilon_{r}$ [see Fig. 1(a)]. The quality factor $Q$ of a $\mathrm{C}$-folded theoretical scatterer, with a small value of $g$, can be calculated using the following expression presented in [6]:

$$
\frac{1}{Q} \simeq\left(\frac{g}{\lambda}\right)^{2}
$$

Based on the preliminary work [3], an optimization of the geometry of the tag has been done to increase the potential of the tag for the authentication purposes. It has been shown in [5], that the radar cross section (RCS) level increases almost 
linearly with $g$. Therefore, a higher value of $g(=2.5 \mathrm{~mm})$ is taken for the development of the optimized tags. In addition, to enhance the tag sensitivity, four scatterers are coupled to realize one tag (called quad C-folded) operating at a resonance frequency $f_{\mathrm{r}}$ larger than $12 \mathrm{GHz}$. As discussed above, and according to expressions (1) and (2), it is obvious that the response of each C-folded resonator depends on its geometrical parameters $L^{\prime}$ and $g$. During the fabrication process, variations of the dimensions of these geometrical parameters are inevitable within permissible range generally provided by the manufacturer. These non-intended modifications yield to a unique signature for each circuit realized from the same mask, leading in turn to authentication possibilities. To test the ability of a system to detect these random variations induced by fabrication inaccuracy, an initial work consists in voluntarily modifying the dimensions of the C-folded scatterer to quantify the minimum variations observable. For this purpose, three groups of five identical chipless quad C-folded tags referenced as N, R20, and R50 are realized. The group $\mathrm{N}$ exhibits the nominal geometrical dimensions. We purposely shortened the length of the arms $L^{\prime}$ by $20 \mu \mathrm{m}$ for R20 group and by $50 \mu \mathrm{m}$ for R50 group. Again, the reason behind such purposely applied variations is to ensure dimensional changes in fabricated prototypes, where the actual randomness happening in the fabrication process is considered as a part of the purposely applied variations. A photograph of the first tag of each group and their dimensions are shown in Fig. 1(a).

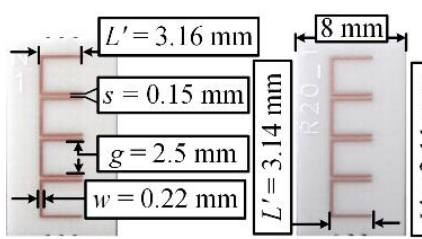

(a) (b)

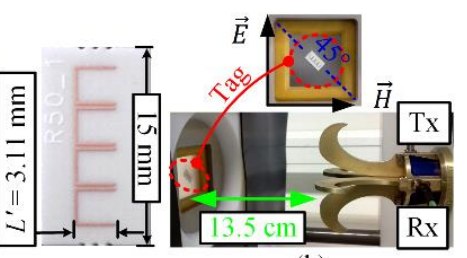

Fig. 1. (a) Photograph of the first tag of each group and their dimensions. (b) Measurement setup in a realistic outdoor environment.

The measurements are done in a monostatic crosspolarization configuration in a realistic outdoor environment by placing the tag at an angle of $45^{\circ}$ to generate a cross response, as shown in Fig. 1(b). The SATIMO QH2000 (2 $32 \mathrm{GHz}$ ) horn antenna with a distance of $13.5 \mathrm{~cm}$ from the tag is used. The experimental results are measured using a vector network analyzer (Agilent Technologies PNA 5222A) with a source power of $0 \mathrm{dBm}$. The measured quantity is the transmission coefficient $S_{21}$. All the five tags from each group are successively measured five times, each measurement is done by removing and repositioning the tag at its position. Furthermore, background normalization is carried out by subtracting an instantaneous measurement taken in the absence of the tag from the measurement taken in the presence of the tag. Fig. 2 shows the measured $S_{21}$ of the first tag of group N. Time windowing [3], is employed to extract the antenna mode with a time window of $10 \mathrm{~ns}$, where $2.2 \mathrm{~ns}$ of early part of the signal is discarded to neglect the structural mode.

In [5], a low pass filter model is used as a first step towards the analytical model for a single C-folded scatterer. This model takes into account both the structural and antenna modes. On the other hand, if the structural mode is neglected by discarding the early part of time domain (TD) response, a second order band pass filter model can be used to model such kind of C-folded scatterer:

$$
T(\omega)=G\left[\frac{\frac{2 m j \omega}{\omega_{o}}}{1+\frac{2 m j \omega}{\omega_{o}}+\left(\frac{j \omega}{\omega_{o}}\right)^{2}}\right]
$$

where, $\omega_{o}$ and $m$ correspond to angular frequency of resonance and damping factor of a pole of second order, respectively. The gain $G$ determines the amplitude level of the signal backscattered by the scatterer. In this way, this model only considers the antenna mode of the backscattered field of a scatterer. Fig. 2(a) exhibits a good agreement between the windowed frequency domain (FD) response (i.e., antenna mode of the C-folded scatterer) and the FD response generated by the analytical model (3). For sake of simplicity, the responses are normalized $(G=1)$. This result validates the proposed model, the experimental acquisition, and timewindowing processes, which are performed systematically for the calculation of the $Q$ factor and the resonance frequency $f_{\mathrm{r}}$. of the resonant tag. For example, the extracted values for the measured response shown in Fig. 2 are $Q_{\mathrm{m}}=92.72$ and $f_{\mathrm{rm}}=12.69 \mathrm{GHz}$. The damping factor $m=1 /\left(2 Q_{\mathrm{m}}\right) \approx 5.39 \times 10^{-3}$.

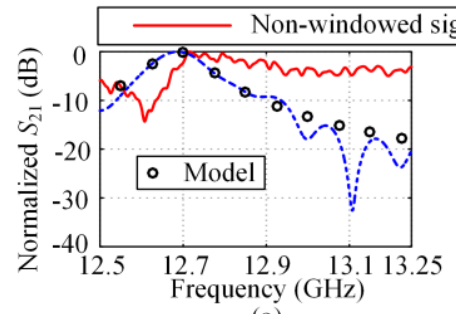

(a)

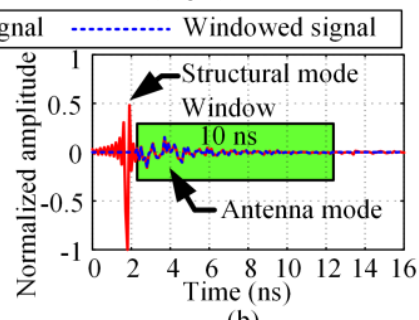

(b)
Fig. 2. Transmission coefficient $S_{21}$ of the first tag of group $\mathrm{N}$ in (a) the frequency domain responses, and in (b) the time domain responses.

\section{SimiLARITY ANALYSIS AND MiCROSCOPIC ANALYSIS}

To highlight the potential of the mathematical model (3) for authentication, a 2D map of similarity is presented in Fig. 3(a), where cosine similarity (CS) function [3] is used to compare the complex FD signals generated by the model. For this comparison, a reference signal with given values of $f_{\mathrm{r} 0}$ and $Q_{0}$ is compared with others, obtained by varying these two parameters $\left(f_{\text {rv }}\right.$ and $\left.Q_{\mathrm{v}}\right)$. Subsequently, CS score is plotted versus $\Delta f_{\mathrm{r}}$ and $\Delta Q$ corresponding to the $f_{\mathrm{rv}}$ and $Q_{\mathrm{v}}$ variations with reference to the $f_{\mathrm{r} 0}$ and $Q_{0}$. From this specific case [Fig. 3(a)] in which the signals have a $Q$ between 80 and 180, it can be observed that as the signals are changing in terms of $f_{\mathrm{rv}}$, dissimilarity increases with a steeper slope in comparison to the change in parameter $Q_{\mathrm{v}}$. Furthermore, to demonstrate that the purposely applied variations among all the groups $\mathrm{N}$, R20, and R50 would be notable to discriminate them from one to another, the extracted $f_{\mathrm{rm}}$ and $Q_{m}$ of all the twenty-five measurements of each group are superimposed for comparison [Fig. 3(a)]. The mean-to-mean variations of the extracted $f_{\text {rm }}$ and $Q_{m}$ between the groups $\mathrm{N}$ and R20 are $\Delta f_{\mathrm{rm}(\mathrm{NR} 20)}=85.3 \mathrm{MHz}$ and $\Delta Q_{\mathrm{m}(\mathrm{NR} 20)}=9.4$, respectively. These 
variations produce a change in cosine similarity $\Delta \mathrm{CS}_{\mathrm{NR} 20}=0.17$. In the same manner, the mean-to-mean variations of the extracted $f_{\mathrm{rm}}$ and $Q_{\mathrm{m}}$ between the groups R20 and $\mathrm{R} 50$ are $\Delta f_{\mathrm{rm}(\mathrm{R} 20 \mathrm{R} 50)}=86.6 \mathrm{MHz}$ and $\Delta Q_{\mathrm{m}(\mathrm{R} 20 \mathrm{R} 50)}=0.6$, which involve a change in cosine similarity $\Delta \mathrm{CS}_{\mathrm{R} 20 \mathrm{R} 50}=0.18$.

To confirm the existence of the dimensional variations along the $L^{\prime}$ in the fabricated prototypes, we carefully measured the $L^{\prime}$ of each arm of each tag by digital microscope images. The resolution of the system is $3 \mu \mathrm{m}$ per pixel. Edge detection technique [see the inset of Fig. 3(b)] is used to measure the dimensions. Fig. 3(b) shows the measured arms length $L_{\mathrm{m}}^{\prime}$ in comparison to the actual value $L^{\prime}$ for all three groups, where the contribution of the natural randomness can be observed. Since all tags were fabricated on one piece of substrate, the effect of randomness is correlated among the tags. For all three groups (N, R20, and R50) the mean values of the $L_{\mathrm{m}}^{\prime}$ distributions are $3.156 \mathrm{~mm}, 3.134 \mathrm{~mm}$, and $3.106 \mathrm{~mm}$, respectively. While the actual values $L^{\prime}$ for all three groups (N, R20, and R50) are $3.16 \mathrm{~mm}, 3.14 \mathrm{~mm}$, and $3.11 \mathrm{~mm}$, respectively. It is clear that for all three groups the mean values of the $L_{\mathrm{m}}^{\prime}$ distributions are less than their corresponding expected values $L^{\prime}$. According to Fig. 3(b), the margin between the $L_{\mathrm{m}}^{\prime}$ distributions of $\mathrm{N}$ and R20 is $3.2 \mu \mathrm{m}$, while the margin between the $L_{\mathrm{m}}^{\prime}$ distributions of R20 and $\mathrm{R} 50$ is $8.3 \mu \mathrm{m}$.

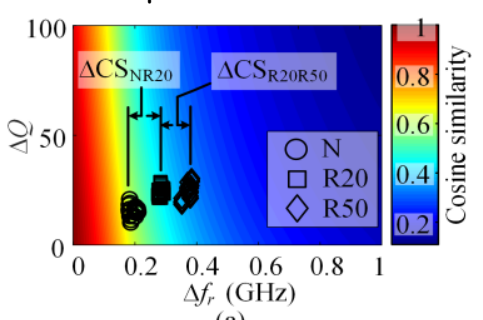

(a)

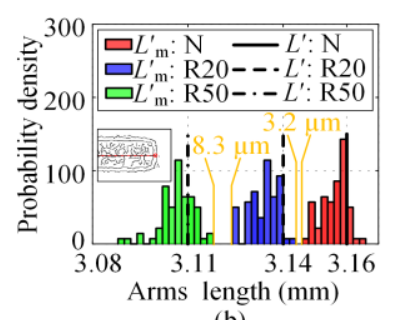

(b)
Fig. 3. (a) $2 \mathrm{D}$ map presenting the cosine similarity obtained by comparing a reference complex FD signal $\left(f_{\mathrm{r} 0}=12.5 \mathrm{GHz}\right.$ and $\left.Q_{0}=80\right)$, with a varying theoretical complex FD signal whose parameters are comprised in the following range: $12.5 \mathrm{GHz} \leq f_{\mathrm{rv}} \leq 13.5 \mathrm{GHz}$ and $80 \leq Q_{\mathrm{v}} \leq 180$. (b) Measured arms length $L_{\mathrm{m}}^{\prime}$ for all groups in comparison to the theoretical arms length $L^{\prime}$. Also, an edge image of the beginning part of the upper arm of the first $\mathrm{C}$ folded scatterer of the first tag of group $\mathrm{N}$.

\section{AUTHENTICATION RESULTS AND COMPARISON}

Considering one group, for instance the $\mathrm{N}$ group, the total number of measurements is 25 (each group is constituted of five tags measured five times each). Therefore, we deduced the number of inter-group combinations among two different groups: $C_{2}^{50}-2\left(C_{2}^{25}\right)=625$. For intra-group, it is found that total number of combinations to compare all measurements within a specific group is $C_{2}^{25}=300$. For all possible combinations, the measurements are compared in the form of complex windowed FD signals using the cosine similarity function. Fig. 4 illustrates the intra-group and inter-group CS distributions. The margin between the maximum inter-group CS and the minimum intra-group CS is about 0.065. The fitted Gaussian probability density functions are used to calculate the probability of false positive (PFP) and the probability of false negative (PFN). We found a probability of error (PE) lower than $10^{-3}$, when the closest distributions are chosen (i.e., worst case): inter-group R20 vs R50 distribution and intra-group R50 distribution (see the inset of Fig. 4). A comparison between the measurements and the model (3) is also presented in Fig. 4. To calculate the model results, average values of extracted $f_{\mathrm{rm}}$ and $Q_{\mathrm{m}}$ of all the 25 measurements of each group, are used to generate a complex FD signal and subsequently signals are compared among the groups. The agreement of the theoretical results with the experimental ones is good. It shows that it is possible to discriminate the tags in a realistic environment merely by focusing on independent quantities $f_{\mathrm{rm}}$ and $Q_{\mathrm{m}}$.

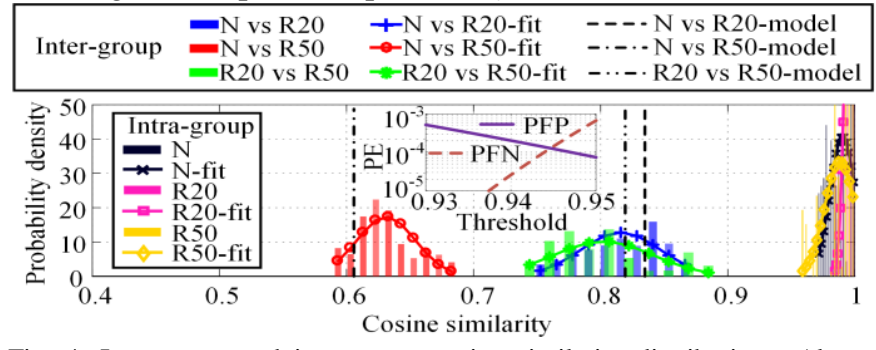

Fig. 4. Intra-group and inter-group cosine similarity distributions. Also, a comparison between the theoretical model and the experimental results. In the inset, error rates between inter-group R20 vs R50 distribution and intra-group R50 distribution.

\section{CONCLUSION}

In this paper randomness along the geometric dimensions of a C-folded resonator were analyzed by a second order band pass filter model. The concept was proved by fabricating three groups of tags (quad C-folded scatterers tags) exhibiting distinct arms length, to account for randomness due to fabrication process. By utilizing cosine similarity as a resemblance metric, it was demonstrated that two groups of the tags exhibiting measured mean variation of around $25 \mu \mathrm{m}$ along the arms length, can be discriminated with a success rate greater than $99.9 \%$ even in a realistic outdoor environment. A good agreement was shown between the theoretical model and the experimental results. According to the validated model proposed in this article, the proposed discrimination technique could potentially differentiate two close structures whose dimensions would vary in the accuracy range of classical manufacturing technique used to realized cheap tags.

\section{REFERENCES}

[1] V. Deepu, A. Vena, E. Perret, and S. Tedjini, "New RF identification technology for secure applications," in IEEE International Conference on RFID-Technology and Applications, Guangzhou, China, 2010, pp. 159-163.

[2] K. Yang, D. Forte, and M. M. Tehranipoor, "UCR: An unclonable chipless RFID tag," in IEEE International Symposium on Hardware Oriented Security and Trust (HOST), Washington, USA, 2016, pp. 7-12.

[3] Z. Ali et al., "On the Uncertainty Analysis of Chipless RFID Approach for Tag Discrimination," submitted in IEEE Microw. Wirel. Compon. Lett.

[4] O. Rance, R. Siragusa, P. Lemaitre-Auger, and E. Perret, "Toward RCS Magnitude Level Coding for Chipless RFID," IEEE Trans. Microw. Theory Tech., vol. 64, no. 7, pp. 2315-2325, Jul. 2016.

[5] A. Vena, E. Perret, and S. Tedjini, "Chipless RFID Tag Using Hybrid Coding Technique," IEEE Trans. Microw. Theory Tech., vol. 59, no. 12, pp. 3356-3364, Dec. 2011.

[6] S. Gov and S. Shtrikman, "On isotropic scattering of electromagnetic radiation," in Eighteenth Convention of Electrical and Electronics Engineers, Israel, 1995, p. 2.4.4/1-2.4.4/5. 